\title{
An electrical model for the Korteweg-de Vries equation
}

S. Giambò, P. Pantano, and P. Tucci

Citation: American Journal of Physics 52, 238 (1984); doi: 10.1119/1.13685

View online: https://doi.org/10.1119/1.13685

View Table of Contents: https://aapt.scitation.org/toc/ajp/52/3

Published by the American Association of Physics Teachers

\section{ARTICLES YOU MAY BE INTERESTED IN}

A comment on the relations between Einstein and Hilbert

American Journal of Physics 52, 206 (1984); https://doi.org/10.1119/1.13691

On the recurrence phenomenon of a resonant spring pendulum

American Journal of Physics 52, 219 (1984); https://doi.org/10.1119/1.13696

The role of induced emf's in simple circuits

American Journal of Physics 52, 208 (1984); https://doi.org/10.1119/1.13692

Proof of "Birkhoff's theorem" in electrodynamics

American Journal of Physics 52, 255 (1984); https://doi.org/10.1119/1.13934

QUANTUM MEASUREMENTS

American Journal of Physics 85, 5 (2017); https://doi.org/10.1119/1.4967925

Magnetic dipole moment measurement

American Journal of Physics 52, 248 (1984); https://doi.org/10.1119/1.13698 


\title{
An electrical model for the Korteweg-de Vries equation
}

\author{
S. Giambo \\ Istituto di Matematica, Via C. Battisti, Messina, Italy \\ P. Pantano \\ CSET (Center for Science Education and Teaching), Department of Physics, University of Calabria, \\ Arcavacata di Rende, 87030 Castiglione, Cosenza, Italy \\ P. Tucci \\ CSET, Department of Physics, University of Calabria, Arcavacata di Rende, 87030 Castiglione, Cosenza, Italy
}

(Received 25 March 1983; accepted for publication 15 July 1983)

In this paper we describe an electrical network, whose current evolution does agree with a Korteweg-de Vries equation. Our aim is to prepare pupils to understand the analytical aspects of nonlinear and dispersive phenomena, which very often are neglected in high-school and graduate textbooks. Some historical remarks introduce the topic and a bibliography is provided.

\section{INTRODUCTION}

Understanding of natural phenomena whose behavior is described by partial differential equations, with nonlinear, dispersive, and dissipative terms, is a problem not easy to solve, because of the difficult mathematical machinery. Yet most natural phenomena do agree with such laws: one of these is the phenomenon of the solitary wave. This is a wave consisting of a single elevation which travels for a considerable distance along a uniform canal, with little or no change of type. J. S. Russell was the first to notice this phenomenon. We was a successful engineer and an odd figure of a Victorian scientist; during his research on the possibility of improving the shape of ships for faster navigation, he realized the inadequacy of that part of theoretical hydrodynamics which refers to the resistance given by fluids to movement of floating bodies. Experiments were carried out on a large and costly scale. It was during these experiments, therefore in a not at all fortuitous way, that J. S. Russell “...observed one very singular and beautiful phenomenon, which is so important, that I shall describe minutely the aspect under which it first presented itself. I happened to be engaged in observing the motion of a vessel at a high velocity, when it was suddenly stopped, and a violent and tumultuous agitation among the little undulations which the vessel had formed around it, attracted my notice. The water in various masses was observed gathering in a heap of a welldefined form around the centre of the length of the vessel. This accumulated mass, raising at last a pointed crest, began to rush forward with considerable velocity towards the prow of the boat, and then passed away before it altogether, and retaining its form, appeared to roll forward alone along the surface of the quiescent fluid, a large, solitary, progressive wave." 1

From the Korteweg-de Vries equation ${ }^{2}$ we know that the phenomenon lies on the balance of two contrary effects: nonlinearity and dispersion. In a nonlinear medium a wave, as it advances, becomes steeper in front and less steep behind; in other words the wave becomes a shock wave. A dispersive medium, instead, flattens the shallow water wave. Water is both a nonlinear and a dispersive medium so a water wave, if properly started, can travel undisturbed.

Many attempts were performed to increase the field of application of classical theory of long waves to make it consistent with the solitary wave phenomenon. But the approximation of calculation was such that some information was lacking about one aspect of the phenomenon-nonlin- earity of the medium-which, with dispersion, is the principal feature of the solitary wave. ${ }^{3}$

In this paper we will show a very easy electrical network whose equation of current evolution is just a Korteweg-de Vries equation. Our aim is to achieve these goals: (1) Pupils can familiarize themselves with nonlinear and dispersive phenomena so that they are more prepared to search out analytical aspects of these phenomena. (2) We can see a soliton on the screen of an oscilloscope connected with the network. (3) This electrical network can be used as an analogical computer in order to find solutions of equations whose analytical integration is too difficult to achieve or whose numerical integration needs too long a computation time.

The second goal is very interesting from a science education and a teaching point of view: we know that two solitary waves travel completely uninfluenced in shape, width, and speed after their interaction. For that Zabusky and Krus$\mathrm{kal}^{4}$ called them "solitons," a word which evokes their behavior as a particle, as well as a wave. "What the computer results showed was that these solitary waves would 'interpenetrate' each other (in two's or three's or more) and emerge from their nonlinear interaction entirely unaffected in shape, amplitude and velocity. One usually thinks of waves being broken up or scattered by nonlinear effects, so the persistence of the waves in this case (dramatically prominent in the computer generated moving picture output) was surprising and noteworthy. (...). Since each solitary wave ever present in a solution of the Korteweg-de Vries equation remained always present, at least 'innately' (in the sense that though it might temporarily disappear from view, by merging in a complicated interaction with one or more others, it would identifiably reappear later), Zabusky and I felt that such waves partook sufficiently of the nature of material particles to justify a particle-like name, reminiscent of the physicists' particles (proton, neutron, electron) and quasiparticles (photon, graviton). We called them solitons." 4 Therefore, in this way, the waveparticle dualism arises in a very classical context-the hydrodynamics - dualism which very often is stressed only in a quantum theory context.

\section{ELECTRICAL MODEL FOR KORTEWEG-DE VRIES EQUATION}

Let us consider the simple network in Fig. 1, consisting of a ladder-type $L C$ circuit containing an inductor $L_{0}$ and a 


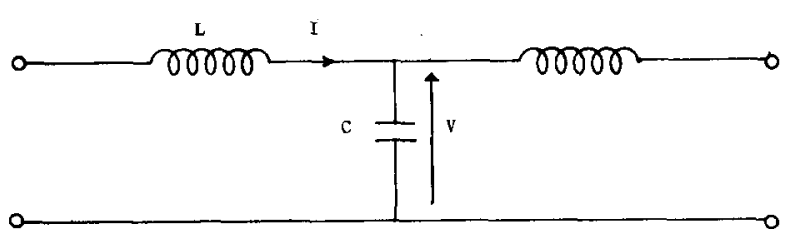

Fig. 1. The elementary network. The connection in series of about 100 of these elementary networks allow us to use for the evolution equation the continuum approximation.

capacitor $C_{0}$, both constant. The equations of propagation are

$$
\begin{aligned}
& \Delta V=-L_{0} \Delta x \frac{\partial I}{\partial t}, \\
& \Delta Q=-C_{0} \Delta x V,
\end{aligned}
$$

where $I$ is the current and $V$ is the voltage. In the continuum approximation from (1) we can derive the wave equation

$$
\frac{\partial^{2} I}{\partial t^{2}}-\frac{1}{L_{0} C_{0}} \frac{\partial^{2} I}{\partial x^{2}}=0
$$

The solutions of (2), as it is well known, are progressive waves

$$
I=\phi\left(x-\frac{1}{\sqrt{L_{0} C_{0}}} t\right)+\phi^{\prime}\left(x+\frac{1}{\sqrt{L_{0} C_{0}}} t\right) .
$$

They represent localized pulses, whose velocity is $1 / \sqrt{L_{0} C_{0}}$, which travel with the same shape during the propagation. This shape is, in general, destroyed when nonlinear or dispersive components are in the network. In fact, if we consider the network in Fig. 2, where now the capacitor $C$ is nonlinear, and the inductor $L$ has a dispersive capacitor in parallel $C_{s} / \Delta x$, propagation in the network occurs in a different way. The fundamental equations are

$$
\begin{aligned}
& \Delta V=-L_{0} \Delta x \frac{\partial I_{1}}{\partial t}, \\
& I_{2}=-C_{s} \frac{\partial}{\partial t} \frac{\Delta V}{\Delta x}, \\
& \Delta Q=-\Delta x\left(C_{0} V-C_{N} V^{2}\right),
\end{aligned}
$$

where $I_{1}$ is the current in the inductor and $I_{2}$ is the current in the dispersive capacitor $C=C_{0}-C_{N} V$. The equation, in the continuum approximation, becomes

$$
\frac{\partial V_{1}}{\partial \tau}+\frac{C_{N}}{C_{0}} V_{1} \frac{\partial V_{1}}{\partial x}+\frac{C_{s}}{2 C_{0}} \frac{\partial^{3} V_{1}}{\partial x^{3}}=0,
$$

where $\tau$ is a contracted time and $x$ is the linear phase of the

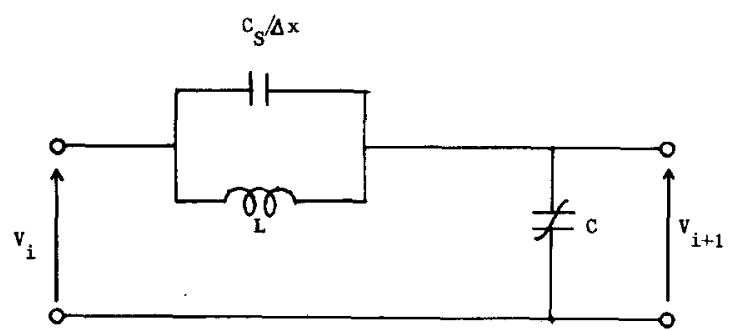

Fig. 2. Elementary network with nonlinear and dispersive components.

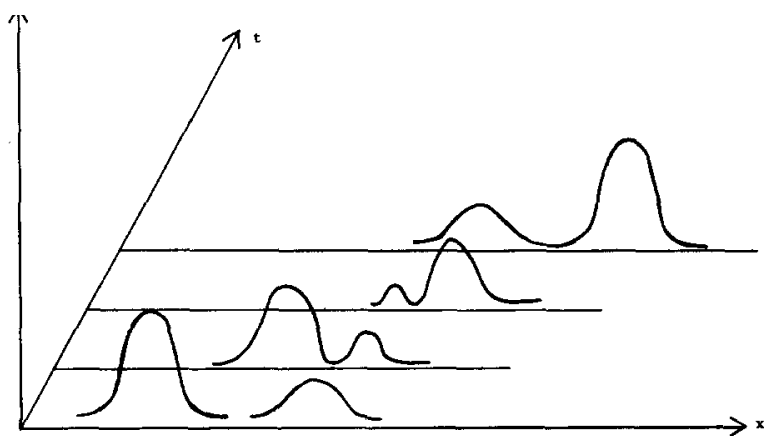

Fig. 3. After their nonlinear interaction two solitons emerge unaffected in shape, amplitude, and velocity.

wave; $V_{1}$ is the first-order perturbation for the voltage (see Appendix A)..$^{5}$

The equation describes the propagation of large wavelength and small amplitude waves in a system which travels with velocity $1 / \sqrt{L_{0} C_{0}}$. Equation (5) is the well-known Korteweg-de Vries equation (KdV). ${ }^{6}$ Typical values of the characteristic of the network are:

$$
\begin{aligned}
& L \Delta x \simeq 0.1 \mu \mathrm{H}, \\
& C_{s} \Delta x \simeq 100 \mathrm{pF}, \\
& \left.C_{N} \Delta x\right|_{V=0} \simeq 500 \mathrm{pF}, \\
& \Delta x=1 \mathrm{~cm}, \\
& V \simeq 5 \mathrm{~V} .
\end{aligned}
$$

The $C$ elements can be obtained with the reversed biased varactor diode, ${ }^{7}$ for example the ITT diode type BA 163. The diode junction capacitance is approximately $100 \mathrm{pF}$ at 3-V reverse bias. Equation (5) exhibits a balance between the nonlinear term $\left(C_{N} / C_{0}\right) V_{1}\left(\partial V_{1} / \partial x\right)$ (see Appendix B) and the dispersive term $\frac{1}{2}\left(C_{s} / C_{0}\right)\left(\partial^{3} V_{1} / \partial x^{3}\right.$ ) (see Appendix C). ${ }^{9}$ In the presence of a localized INPUT, we can observe particular pulses-the solitons-which travel in the network as progressive waves. Their velocity $u$ and width $S_{0}$ depend, of course, on the amplitude $A_{0}$ by means of the relations:

$$
\left.u\right|_{\text {soliton }}=\left(1+\frac{C_{N} A_{0}}{3 C_{0}}\right) \frac{1}{\sqrt{L_{0} C_{0}}} \text { and } S_{0}^{2} \propto A_{0}^{-1} \text {. }
$$

Their shape can be represented by a soliton-like solution of

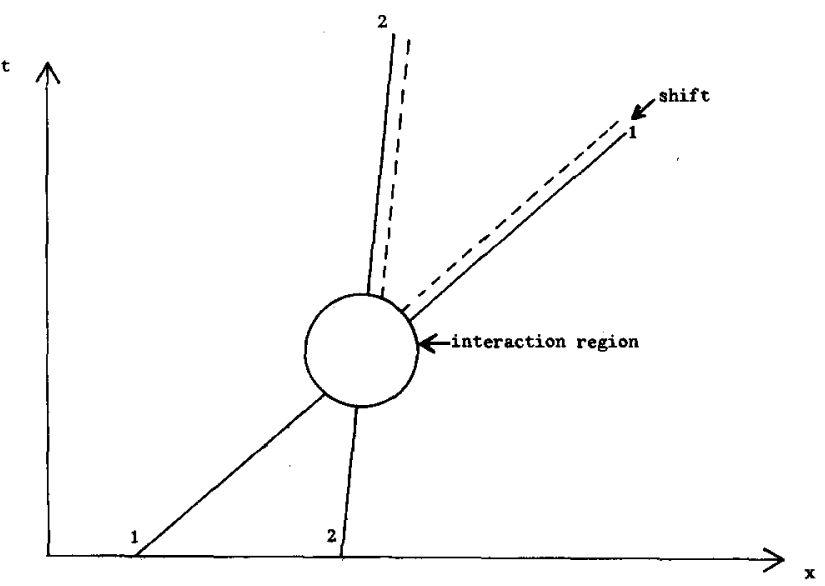

Fig. 4. Phase shift after the interaction of two solitons. 


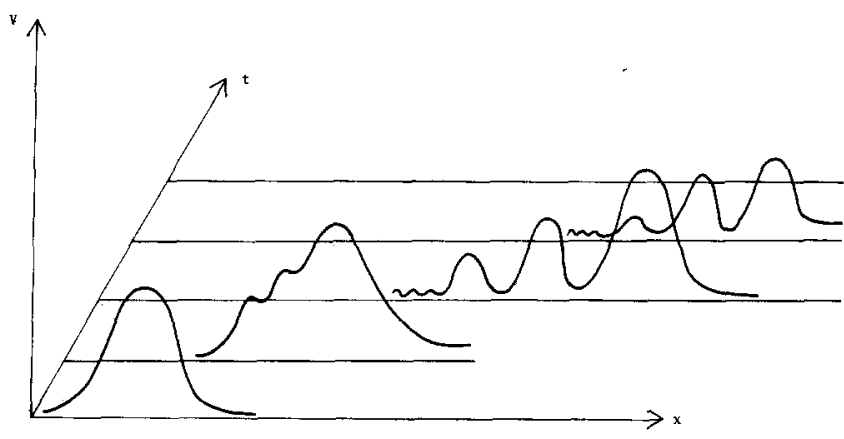

Fig. 5. An input decomposes itself into a series of solitons plus a dispersive vanishing part.

Eq. (5):

$$
V_{1}=A_{0} \operatorname{sech}^{2}\left[\sqrt{\frac{C_{N} A_{0}}{6 C_{s}}}\left(x-\frac{C_{N} A_{0}}{3 C_{0}} \tau\right)\right] .
$$

If we have two different solitons amplitude (see Fig. 3), we observe that their interaction is nonlinear and after the interaction they come out with the same shape: only a phase shift can be observed (see Fig. 4). Two other interesting effects can be observed in the network: the decomposition of any input into a series of solitons plus a dispersive vanishing part (Fig. 5) (see Appendix D) and the phenomenon of recurrence. ${ }^{10}$ The last phenomenon is the return of the initial input in the initial state after a certain time.

Finally we can note that a similar approach to visualize the nonlinear characteristic of the solitons allows us a very interesting introduction of advanced items of research. In fact, if we modify the nonlinear dispersive network in Fig. 2, with the three-dimensional network in Fig. 6 being the new configuration, the propagation does agree with the following modified $\mathrm{KdV}$ equation:

$$
\begin{aligned}
& \frac{\partial V_{1}}{\partial r}+\frac{1}{\sqrt{L_{0} C_{0}}}\left(1-\frac{C_{N} V_{1}}{C_{0}}\right) \frac{\partial V_{1}}{\partial t} \\
& \quad-L_{0} C_{0} \frac{1+\cos ^{2} 2 \varphi}{48}-\frac{\partial^{3} V_{1}}{\partial t^{3}}+\frac{V_{1}}{2} \frac{d}{d r} \ln \Delta=0,
\end{aligned}
$$

where $r$ is now a radial coordinate and $(d / d r) \ln \Delta$ takes into account the geometrical effects on the propagation.

The relationship between amplitude, width, and velocity are the same as in (6) but now they depend on the radial coordinate. Currently an analytical method for solving Eq. (8) does not exist which permits us to investigate this prob-

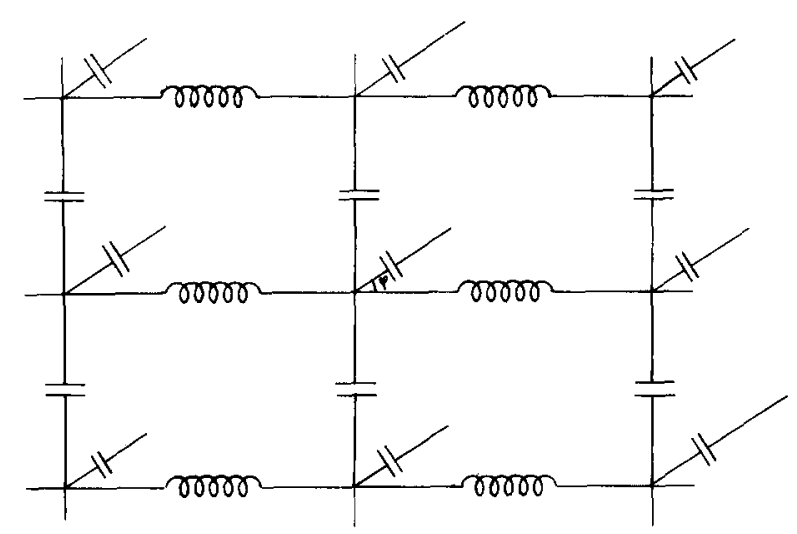

Fig. 6. Nonlinear, dispersive three-dimensional network. lem, in the same way that the Inverse Scattering Transform allows us to investigate the Cauchy problem. Therefore, the three-dimensional network can be used as an analogic computer. We note, in conclusion, that the components of the described networks have a constant characteristic: if we leave out this restriction, by thinking a space or time dependence, a new series of equations arises and new phenomena can be observed. ${ }^{11}$ Other Electrical Analog circuits have been used to study many different phenomena. ${ }^{12}$

\section{APPENDIX A: DERIVATION OF THE KdV EQUATION}

From Eq. (1) we can derive the following equation:

$$
\frac{\partial^{4} V}{\partial x^{2} \partial t^{2}}+\frac{1}{L_{0} C_{s}} \frac{\partial^{2} V}{\partial x^{2}}-\frac{1}{C_{s}} \frac{\partial^{2} Q(V)}{\partial t^{2}}=0 .
$$

If we put $Q(V)=C_{0} V-C_{N} V^{2}$ and $T=t / \sqrt{L_{0} C_{0}}$ Eq. (A1) becomes

$$
\frac{C_{s}}{C_{0}} \frac{\partial^{4} V}{\partial x^{2} \partial T^{2}}+\frac{C_{N}}{C_{0}} \frac{\partial^{2}\left(V^{2}\right)}{\partial T^{2}}-\frac{\partial^{2} V}{\partial T^{2}}+\frac{\partial^{2} V}{\partial x^{2}}=0 .
$$

Let us consider now the following asymptotic expansion around the state $V_{0}=0$ :

$$
V=\epsilon V_{1}+\epsilon^{2} V_{2}+\ldots \text {, }
$$

where $\epsilon$ is a small parameter. Now we introduce the stretched variables ${ }^{5}$

$$
\begin{aligned}
& x=\epsilon^{1 / 2}(x-T), \\
& \tau=\epsilon^{3 / 2} T .
\end{aligned}
$$

Therefore, if we put (A3) and (A4) into (A2) and if we make the coefficients of the series in $\epsilon$ equal to zero, we obtain at lowest order

$$
\frac{\partial V_{1}}{\partial \tau}+\frac{C_{N}}{C_{0}} V_{1} \frac{\partial V_{1}}{\partial x}+\frac{C_{s}}{2 C_{0}} \frac{\partial^{3} V_{1}}{\partial x^{3}}=0
$$

This is Eq. (5).

\section{APPENDIX B: NONLINEAR FEATURES OF THE EQUATION}

If we consider an initial localized pulse

$$
V_{1}(x, 0)=f(\xi)
$$

where $\xi=x$ at $t=0$, we can consider the characteristic curves

$$
\frac{\partial x}{\partial \tau}=\frac{C_{N}}{C_{0}} V_{1}
$$

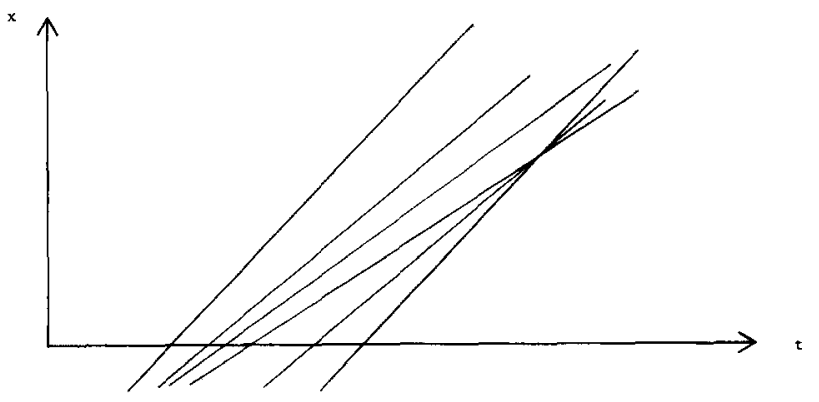

Fig. 7. Characteristic curves whose angular coefficient is $C_{0} / C_{N} 1 / f(\xi)$. 


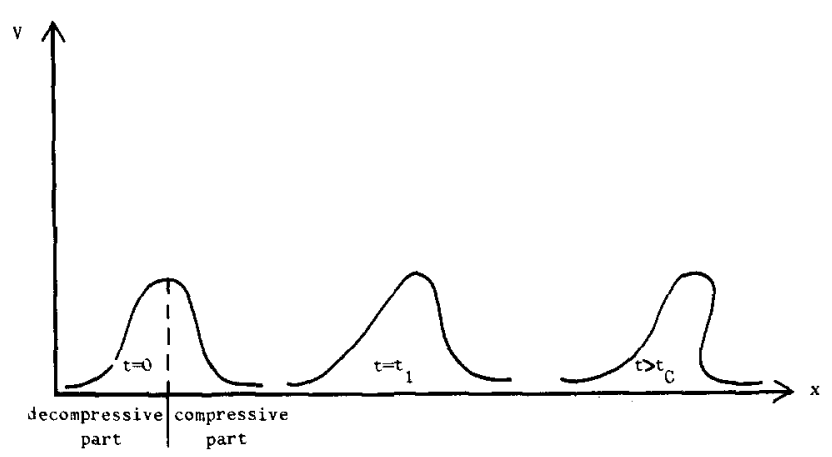

Fig. 8. Because of the fact that greater is the quote, faster is the travel, after the time $t_{C}=+\min 1 /\left|C_{N} / C_{0} f(\xi)\right|$ the pulse decays in a shock wave.

and we can observe that $V_{1}$ is constant on these curves, because $V_{1}$ satisfies Eq. (B1). At this stage we do not know these curves. For a given point $(x, 0)$ there is a characteristic curve which passes through. The angular coefficient of this curve is $\left(C_{0} / C_{N}\right)[1 / f(\xi)]$. The curve equation is

$$
x=\left(C_{N} / C_{0}\right) f(\xi) \tau+\xi \text {. }
$$

We can observe that the greater quotes travel with greater velocities. (See Figs. 7 and 8.) Therefore the shape is modified during the propagation and at the time

$$
t_{C}=+\min \frac{1}{\left|C_{N} / C_{0} f^{\prime}(\xi)\right|} \text { with } f^{\prime}<0
$$

the function $V_{1}$ is multivalued, i.e., after $t_{C}$ the pulse decays in a shock wave.

\section{APPENDIX C: DISPERSIVE SYSTEM}

In order to describe how a dispersive system works, let us make the KdV equation linear. The general solution of a linear equation is

$$
\Phi(x, t)=\frac{1}{2 \pi} \int_{-\infty}^{+\infty} \chi(K) \exp \{i[K x-\omega(K) t]\} d K
$$

where $\chi(K)$ is the Fourier component of the initial perturbation

$$
\chi(K)=\int_{-\infty}^{+\infty} \Phi(x, 0) e^{-i K x} d x
$$

If we consider the asymptotic form of (C1) for large values of $t$, and if we use the stationary phase method, we may readily derive

$$
\begin{aligned}
& \Phi(x, t)=\sqrt{\frac{2}{\pi\left|\omega^{\prime \prime}(K)\right| t}} \\
& \times \operatorname{Re}\{\mathcal{X}(K) \exp [i(K x-\omega(K) t \\
& \left.\left.\left.-(\pi / 4) \operatorname{sign} \omega^{\prime \prime}(K)\right)\right]\right\} \text {, }
\end{aligned}
$$

where $K$ is the positive root of the equation,

$$
u(K)=\omega^{\prime}(K)=x / t \text {. }
$$

The energy decays as $|\chi(K)|^{2} /\left|\omega^{\prime \prime}(K)\right| t$ and localized initial data are spread on all the space. This happens also for the linearized $\mathrm{KdV}$ equation.

\section{APPENDIX D; INVERSE SPECTRAL TRANSFORM}

First of all we transform Eq. (5) in $U_{t}-U U_{x}+U_{x x x}=0$, with $U=-2 C_{N} / C_{S} V_{1}$ and $t=C_{S} / 2 C_{0}$. To this last equation we apply the rule of the

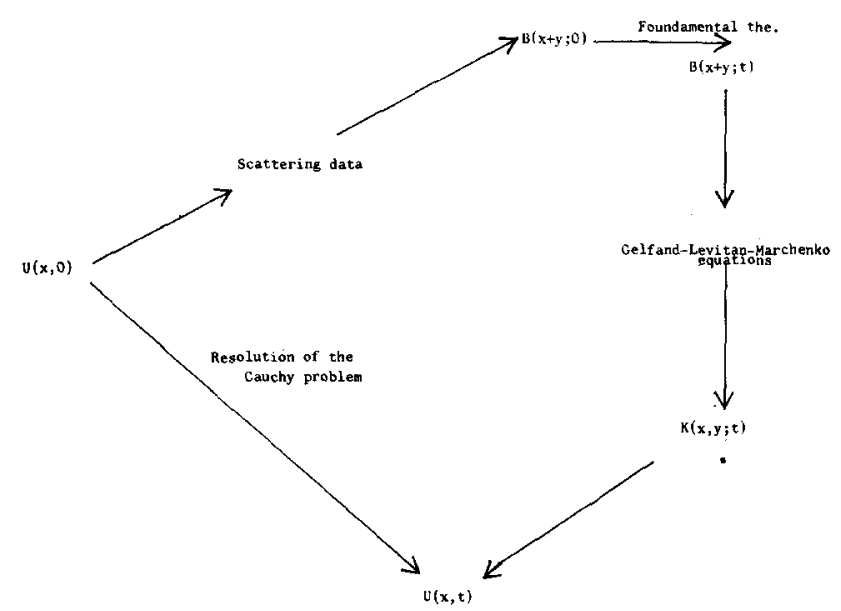

Fig. 9. How we apply the Inverse Spectral Transform to KdV.

Inverse Spectral Transform which is very powerful machinery to solve exactly the Cauchy problem for a very large class of partial differential equations. It can be thought of as the nonlinear correspondence of the Fourier Transform. For the KdV equation it works in this way (Fig. 9).

To an initial value $U(x, 0)$ for the $\mathrm{KdV}$ subject to the condition

$$
\int_{-\infty}^{+\infty}(1+|x|)|u(x, 0)| d x<+\infty
$$

we associate the Schrödinger condition

$$
\psi_{x x}-(u-\lambda) \psi=0
$$

Now we solve the direct spectral problem for (D1), i.e., we deduce (a) the discrete eigenvalues $\lambda_{n}=-K_{n}^{2}, n=1,2, \ldots, N$; (b) the normalitation coefficient $C_{n}$, where

$$
C_{n}=\lim _{x \rightarrow \infty} e^{K_{n} x} \psi_{n}(x)
$$

where $\psi_{n}$ are the corresponding eigenfunctions; (c) the reflection coefficients $b(K)$ for the continuous spectrum $\lambda=K^{2}$, where $b(K)$ is defined by the asymptotic values of $\psi$, i.e.,

$$
\psi(x, 0) \simeq\left\{\begin{array}{l}
e^{-i K x}+b(K) e^{i K x}, \quad x \rightarrow \infty \\
a(K) e^{-i K x}, \quad x \rightarrow-\infty,
\end{array}\right.
$$

where $a(K)$ is the transmission coefficient. The third step is to define the function

$$
B(\zeta) \equiv \sum_{n=1}^{N} C_{n}^{2} e^{-K_{n} \zeta}+\frac{1}{2 \pi} \int_{-\infty}^{+\infty} b(K) e^{i K \zeta} d K
$$

Now we use the following fundamental theorem: if $U(x, t)$ evolves accordingly to the $\mathrm{KdV}$ equation, the scattering data are given by
(a) $\lambda_{n}(t)=\lambda_{n}(0)$
(b) $C_{n}(t)=C_{n}(0) e^{4 K_{n}^{3} t}$,
(c) $b(K, t)=b(K, 0) e^{8 i K^{3} t}$.

Therefore, if the initial scattering data are known, the scattering data are known at all times.

We now write the Gelfand-Levitan-Marchenko equation 
$K(x, y ; t)+B(x, y ; t)+\int_{-\infty}^{+\infty} B(y+z ; t) K(x, z ; t) d z=0$.

We now solve this linear equation for $K(x, y ; t)$; therefore, $U(x, t)$ which satisfies the $\mathrm{KdV}$ initial data $U(x, 0)$ is given by

$$
U(x, t)=-2 \frac{\partial}{\partial x} K(x, X ; t) .
$$

If we consider the initial data

$$
u(x, 0)=-\frac{1}{2} a^{2} \operatorname{sech}^{2}\left\{\frac{1}{2} a x\right\}
$$

we have the single eigenvalue for the scattering data $\lambda=a^{2} / 4$ and $b(K, 0)=0$. Then, if we apply the Inverse Scattering Transform, we have

$$
u(x, t)=-\frac{1}{2} a^{2} \operatorname{sech}^{2}\left\{\frac{1}{2} a\left(x-a^{2} t\right)\right\}
$$

which is the shape of a single soliton. The amplitude is proportional to the velocity and is equal to $-4 \lambda_{n}$. The multisolitons are given for $U(x, 0)$ with $N$ discrete eigenvalues accordingly to $b(k, 0)=0$, and the solution is given by

$$
u(x, t)=-2 \frac{\partial^{2}}{\partial x^{2}} \log \operatorname{det}(I+C),
$$

where $I$ is the $N \times N$ identity matrix and

$$
C \equiv\left\{C_{m}(t) C_{n}(t) \frac{\exp \left[-\left(K_{m}+K_{n}\right) t\right]}{K_{m}+K_{n}}\right\}
$$

In the presence of two discrete eigenvalues we observe an interaction region in the space-time, and after the interaction region only two single pulses. In the interaction region the soliton-like pulses are shifted. If we also have $b(K, 0)$ a decaying dispersive residue is observed which can be neglected after a certain time.

'J. S. Russell, Trans. R. Soc. Edinburgh XIV, 1 (1837). Detailed research on this problem by J. S. Russell is described in J, Robison and J. S. Russell, Rep. Brit. Assn. Adv. Sci. VI, 417 (1838); J. Robison and J. S. Russell, Rep. Brit. Assn. Adv. Sci. XIII, 311 (1845). You can find a biography of J. S. Russell in G. S. Emmerson, John Scott Russell ( John Murray, London, 1977).

${ }^{2}$ D. J. Korteweg and G. De Vries, Phil. Mag. XXXIX, 422 (1895). Many review articles give some information about the history of the Korteweg-De Vries equation from J. S. Russell's notice on the solitary wave. The most important are: R. M. Miura, SIAM Rev. 18, 412 (1976); R. K. Bullough and P. J. Caudrey, "The Soliton and its History," in Solitons, edited by R. K. Bullough and P. J. Caudrey (Springer-Verlag, Berlin, 1980), pp. 1-64; J. W. Miles, J. Fluid Mech. 106, 131 (1981); A. Libchaber and G. Toulouse, La Rèchèrche 7, 1027 (1976); R. K. Bullough and R. K. Dodd, Solitons and Condensed Matter Physics (Springer-Verlag, Berlin, 1978), pp. 2-21; F, van der Blij, Nieuw Archief Voor Wiskunde XXVI, 54 (1978).

${ }^{3}$ Two of the authors of this paper have studied particularly this aspect in P. Pantano and P. Tucci, Proc. $3^{\circ}$ Congress on History of Physics, Palermo, October 1982.

${ }^{4}$ N. J. Zabusky and M. D. Kruskal, Phys. Rev. Lett. 15, 240 (1965).

${ }^{5}$ Equation (5) can be deduced from Eqs. (4) by using the perturbationreduction methods (see Appendix A). See R. Hirota and K. Suzuki, Proc. IEEE 61, 1483 (1973); K. E. Lonngren, in Solitons in Action, edited by K. Lonngren and A. C. Scott (Academic, New York, 1979). These methods were used first by C. S. Gardner and G. K. Morikawa, Rep. Courant Institute, NY0-9082, New York, 1960 and by H. Washimi and T. Taniuti, Phys. Rev. Lett. 17, 996 (1966) to deduce the KdV equation in a collisionless plasma. The general form of these methods is in $T$. Taniuti and C. C. Wei, J. Phys. Soc. Jpn. 24, 941 (1968) and a giustification in term of "long field" in T. Taniuti, Suppl, Prog. Theor. Phys. 55, 1 (1974). The three-dimensional form for dissipative systems is in P. Germain,
Progressive Waves (Centenaire de Prandt1, 1971) and for dispersive systems in S. Giambò, A. Greco, and P. Pantano, C. R. Acad. Sci. Paris 289, 556 (1979).

${ }^{6} \mathrm{The} \mathrm{KdV}$ equation describes a large class of physical phenomena beyond water waves and the electrical networks. The main fields of applications are: (a) ion-acoustic waves in plasma [see $\mathbf{H}$. Washimi and $\mathbf{T}$. Taniuti, Phys. Rev. Lett. 17, 996 (1966); M. O. Tran, Physica Scripta 20, 317 (1979); H. Ikezi, in Solitons in Action (Academic, New York, 1978)]. (b) magnetohydrodynamics waves in plasma [see T. Kawahara, J. Phys. Soc. Jpn. 27, 1331 (1969); T. Kakutani, Suppl. Progr. Theor. Phys. 55, 97 (1974); (c) anharmonics lattices [N. J. Zabuski and M. D. Kruskal, Phys. Rev. Lett. 15, $240(1965)]$ and in general for hyperbolic systems with a small dispersive part (see Ref. 1). This equation was integrated at first by C. S. Gardner, J. M. Greene, M. D. Kruskal, and R. M. Miura, Phys. Rev. Lett. 19, 1095 (1967) and by the same authors in Comm. Pure Appl. Math. 27, 97 (1974) by using the Spectral Transform (see Appendix D). V. E. Zakharov and L. D. Faddeev, Functional Anal. Appl. 5, 280(1972) proved that the $\mathrm{KdV}$ is a completely integrable Hamiltonian system. The existence of quasiperiodic solutions are in P. D. Lax, Comm. Pure Appl. Math. 28, 141 (1975); P. D. Lax, SIAM Rev. 18, 351 (1976). The periodic problem is in B. A. Dubrovin, Funk. Anal. Appl. 9, 61 (1975); S. P. Novikov, Funct. Anal. Appl. 8, 236 (1974). The existence theory is in R. Teman, J. Math. Pures Appl. 48, 159 (1969); Y. Tametaka, Proc. Jpn. Acad. 45, 552, 556 (1969); J. L. Bona and R. Smith, Phil. Trans. R. Soc. London, Ser. A 278, 555(1975). The solitons of the KdV equation can be obtained by using also the Hirota method in R. Hirota, in Backlund Transformation, edited by A. Dold and B. Eckmann (Springer-Verlag, Berlin, 1976); W. Malfliet, Am. J. Phys. 49, 666 (1981). Recently a large variety of review papers or books is published on KdV equation and on the Inverse Scattering Transform. We list some of them: Solitons, edited by G. Eilenberger (Springer-Verlag, Berlin, 1981); Spectral Transform and Solitons: Tools to Solve and Investigate Non-Linear Evolution Equations, edited by F. Calogero and A. Degasperis, to be published; Elements in Solitons Theory, edited by G. L. Lamb, Jr. (Wiley, New York, 1981); The Inverse Scattering Transformation and the Theory of Solitons, edited by W. Eckhars and A. van Arten (North-Holland, Amsterdam, 1981); Asymptotic Method in Non-Linear Wave Theory, edited by A. Jeffrey and T. Kawahara (Pitman, London, 1982); Backlund Transformation, edited by R. M. Miura, Lecture Notes in Mathematics 515 (Springer, New York, 1976); Non-Linear Evolution Equation and Dynamical System, edited by M. Boiti, F. Pempinelli, and G. Soliani, Lecture Notes in Physics 120 (Springer-Verlag, Berlin, 1980); A. C. Scott, F. Y. F. Chu, and D. W. Mclaughlin, Proc. IEEE 51, 1443 (1973); C. Cercignani, Rivista Nuovo Cimento 7, 429 (1977); L. D. Faddeev and V. E. Korepin, Phys. Rep. 42, 1 (1978); M. J. Ablowitz, Stud. Appl. Math. 58, 17 (1978).

${ }^{7}$ R. H. Freeman and A. E. Karbowiak, J. Phys. D Appl. Phys. 10, 633 (1977); R. Hirota and K. Suzuki, see Ref. 5; K. E. Lonngren, see Ref. 5. ${ }^{8}$ G. B. Whitman, Linear and Non-Linear Waves (Wiley, New York, 1974).

${ }^{9}$ Ibidem and R. I. Karpmann, Non-Linear Waves in Dispersive Media (Pergamon, Oxford, 1975).

${ }^{10}$ K. Fukushima, M. Wadati, T. Kotera, K. Sawada, and Y. Narahara, J. Phys. Soc. Jpn. 48, 1029 (1980).

${ }^{11}$ The method of deducing the equation for the case of propagation in the three-dimensional network is similar to the one described in Ref. 5. The following equation was derived, first, by Y. A. Stephaniants, Wave Motion 3, 335 (1981), by using a "linear ray" method developed by L. A. Ostrovsky, Int. J. Non-linear Mech. 11, 416 (1976):

$$
\begin{aligned}
\frac{\partial V}{\partial r} & +\frac{1}{\sqrt{L_{0} C_{0}}}\left(1-\frac{C_{N} V}{C_{0}}\right) \frac{\partial V}{\partial t} \\
& -\frac{1+\cos ^{2} 2 \varphi}{48 / L_{0} C_{0}} \frac{\partial^{3} V}{\partial t^{3}}+\frac{V}{2} \frac{d}{d r} \ln \Delta=0 .
\end{aligned}
$$

This equation is a $\mathrm{KdV}$ with a new geometrical term $(d / d r) \ln \Delta$. A cylindrical symmetry is supposed. This equation can be obtained by using the perturbation-reduction method when no symmetry is imposed. Equation (8) was deduced by S. Maxon and J. Viecelli, Phys. Fluids 17, 1614 (1974) during their research on ion-acoustic waves in a collisionless plasma. The equations similar to (8) describe many physical situations such 
as one-dimensional shallow water waves of variable depth [see T. Kakutani, J. Phys. Soc. Jpn. 30, 272 (1971); R. S. Jonhson, J. Fluid Mech. 60, 813 (1973)]; the propagation in many dimensions of plasma waves [see $P$. Pantano and S. Giambò, Lett. Nuovo Cimento 34, 380 (1982)]; shallow water waves of constant depth [see P. Parasad and R. Revindran, J. Inst. Math. Appl. 20, 9 (1977)]; and many other physical problems [see N. Asano and H. Ono, J. Phys. Soc. Jpn. 31, 1830 (1971)]. This equation can be integrated analytically when $\Delta \propto 2$ [See F. Calogero and A. Degasperis, Lett. Nuovo Cimento 23, 150 (1978); A. Nakamura and H. H. Chen, J. Phys. Soc. Jpn. 50, 711 (1981); J. J. C. Nimmo and D. G. Cryghton, Phys. Lett. 82A, 211 (1981); C. Horrocks and P. Wilkinson,
Phys. Lett. 81A, 305 (1981)] or by using an asymptotic perturbation method [see K. I. Karpman and E. M. Maslov, Sov. Phys. JETP 46, 41 (1977); D. J. Kaup and A. C. Newell, Proc. R. Soc. London, Ser. A 361, 41 (1978); K. Ko and H. H. Khuel, Phys. Fluids 22, 1343 (1979)].

${ }^{12}$ K. Muroya and S. Watanabe, J. Phys. Soc. Jpn. 50, 3159 (1981); S. Watanabe, ibid. 50, 3166 (1981); T. Yagi, ibid. 50, 2737 (1981); P. Pantano, to be published in Lett. Nuovo Cimento (1983); T. Yoshinaga and T. Kakutami, J. Phys. Soc. Jpn. 49, 2072 (1980); F. Kako, ibid. 47, 1686 (1979); T. Yoshinaga and T. Kakutani, ibid. 51, 1303 (1982); T. Yoshinaga, N. Sugimoto, and T. Kakutani. ibid. 50, 2122 (1981); K. Fukushima, M. Widati, and Y. Narahara, ibid. 49, 1593 (1980).

\title{
The spot of Arago: New relevance for an old phenomenon
}

\author{
James E. Harveya) \\ Rockwell International, Rocketdyne Division, Kirtland Air Force Base, New Mexico 87117 \\ James L. Forgham \\ United Technologies of New Mexico, Inc., Albuquerque, New Mexico 87185
}

(Received 14 March 1983; accepted for publication 18 April 1983)

\begin{abstract}
The "spot of Arago" has been a controversial topic since its inception in 1818 when Poisson predicted its existence in an attempt to discredit Fresnel's wave theory of light. Arago performed the experiment and found the surprising prediction was true, thus putting Fresnel's theory on a firm technical foundation. In recent years, the spot of Arago, which exists as a bright spot at the center of the geometrical shadow of a circular obstruction, has caused substantial grief in various high-energy laser applications and has come to be considered more of a nuisance than a curiosity. This paper suggests that the size and shape of the spot of Arago is characteristic of the wave-front aberrations of the incident beam and can therefore be used to advantage as a beam sample for wave-front analysis of annular beams. The implementation of this wave-front sampling scheme would eliminate the requirement for a special beam-sampling optical component and thus reduce to a minimum the deleterious effects upon the beam frequently accompanying the use of such components. Both experimental and numerical results are presented along with a discussion of the capabilities and limitations of this particular beam sample for diagnostic purposes.
\end{abstract}

\section{INTRODUCTION}

In 1818 Fresnel presented his famous essay on the wave theory of light to the French Academy. Poisson, a member of the academy and an advocate of the corpuscular theory of light, deduced from Fresnel's wave theory that a bright spot of light should appear at the center of the shadow of an illuminated disk or circular obstruction. This prediction, he claimed, violated common sense and hence refuted Fresnel's wave theory. However, the prediction was experimentally tested and verified by Arago (another member of the Academy), thus firmly establishing Fresnel's theory and contributing to the rapid demise of the corpuscular theory of light (Poisson's predicted spot had been observed by Maraldi a century earlier but had gone largely unnoticed at the time). Due to Arago's historically important confirmation of Fresnel's wave theory of light we refer to this phenomena as the "spot of Arago" rather than "Poisson's bright spot" which is more popular in the literature.

After this rather dramatic introduction to the scientific community over 150 years ago, the spot of Arago gradually receded into the background as a historically significant but not particularly useful optical phenomenon. R. W. Pohl showed that a photographic objective can be replaced by a steel sphere or an opaque disk; however, this potential application has survived only as a textbook curiosity rather than a practical reality because of low image brightness and poor resolution due to the smoothing effect of the rather prominent ring structure surrounding the spot of Arago.

In recent years the almost-forgotten spot of Arago has made its presence emphatically known in certain annular high-energy laser (HEL) systems by depositing substantial flux levels at inconvenient locations throughout the system. Some attempts to solve this problem by simple shadowing techniques are inherently impossible due to the nature of the diffraction process which produces the spot of Arago.

Since the spot of Arago appears to be an inherent part of annular (obscured) laser beams, this paper is a preliminary attempt to determine its properties in the presence of elementary aberrations and to evaluate its potential value as a beam sample available for wave-front analysis.

\section{TECHNICAL DISCUSSION}

The Rayleigh-Sommerfeld formulation for the diffracted wave field produced by an aperture illuminated by a monochromatic beam is given by 